\title{
FUNDAÇÃO E IMPLANTAÇÃO DE UMA LIGA ACADÊMICA DE ENFERMAGEM EM CARDIOLOGIA
}

\author{
Lucas Teixeira de Sousa Santos \\ Universidade Estadual Vale do Acaraú \\ tei.lucasenfer@gmail.com \\ Kairo Cardoso da Frota \\ Universidade Estadual Vale do Acaraú \\ kairo.enfer@gmail.com
}

\author{
Keila Maria de Azevedo Ponte \\ Universidade Estadual Vale do Acaraú \\ keilinhaponte@hotmail.com \\ Ingrid Kelly Morais Oliveira \\ Universidade Estadual Vale do Acaraú \\ ingridkelly17.ik@gmail.com \\ Fabiene Lima Parente \\ Universidade Estadual do Ceará \\ fabiene.parente@stacasa.com.br
}

\section{Resumo}

Objetivo: Descrever o processo de fundação e implantação de uma liga acadêmica de enfermagem em cardiologia. Método: Trata-se de um relato de experiência acerca do processo de fundação e implantação da Liga de Enfermagem em Cardiologia de uma universidade pública no Ceará. Resultados: A Liga de Enfermagem em Cardiologia emerge do interesse pela cardiologia despertado por estudantes ao longo da graduação em enfermagem, visando proporcionar uma melhor formação profissional a seus integrantes; promover a integração entre acadêmicos e docentes, pesquisadores da saúde cardiovascular, serviço de saúde e população; com atividades no âmbito do tripé: ensino-pesquisa-extensão com vistas a contribuir com a sociedade na promoção da saúde cardiovascular. Conclusões: A criação da liga possibilitou aos acadêmicos autonomia, com aprofundamento em uma área não muito explorada pela grade curricular do curso, garantindo o desenvolvimento de competências, habilidades e atitudes de responsabilidade, liderança, execução do trabalho em equipe e gerenciamento frente a uma atividade extracurricular de ensino-pesquisa-extensão.

Palavras-chave: Educação Superior; Enfermagem Cardiovascular; Relações Comunidade-Instituição.

\section{FOUNDATION AND IMPLEMENTATION OF AN ACADEMIC NURSING LEAGUE IN CARDIOLOGY}

\section{Abstract}

Objective: To describe the process of founding and implementing an academic nursing league in cardiology. Method: This is an experience report about the process of founding and implementing the Cardiology Nursing League of a public university in Ceará. Results: The Cardiology Nursing League emerges from the interest in cardiology aroused by students throughout their undergraduate nursing courses, aiming to provide better professional training to its members; promote integration between academics and teachers, researchers in cardiovascular health, health services and the population; with activities within the scope of the tripod: teaching-research-extension with a view to contributing to society in promoting cardiovascular health. Conclusions: The creation of the league allowed the academics autonomy, with deepening in an area that is not very explored by the curriculum of the course, guaranteeing the development of competences, skills and attitudes of responsibility, leadership, execution of teamwork and management when facing an activity extracurricular teaching-research-extension.

Keywords: Education Higher; Cardiovascular Nursing; Community-Institutional Relations.

\section{FUNDACIÓN E IMPLEMENTACIÓN DE UNA LIGA DE ENFERMERÍA ACADÉMICA EN CARDIOLOGÍA}

\section{Resumen}

Objetivo: describir el proceso de fundación e implementación de una liga académica de enfermería en cardiología. Método: Este es un informe de experiencia sobre el proceso de fundación e implementación de la Liga de Enfermería en Cardiología de una universidad pública en Ceará. Resultados: La Liga de Enfermería en Cardiología surge del interés en la cardiología despertado por los estudiantes a lo largo de sus cursos de pregrado en enfermería, con el objetivo de proporcionar una mejor capacitación profesional a sus miembros; promover la integración entre académicos y docentes, investigadores en salud cardiovascular, servicios de salud y población; con actividades en el ámbito del trípode: enseñanzainvestigación-extensión con miras a contribuir a la sociedad en la promoción de la salud cardiovascular. Conclusiones: La creación de la liga permitió la autonomía académica, con una profundización en un área poco explorada por el plan de estudios del curso, asegurando el desarrollo de competencias, habilidades y actitudes de responsabilidad, liderazgo, ejecución del trabajo en equipo y gestión frente a una actividad. enseñanza extracurricular-investigación-extensión.

Palavras clave: Educación Superior; Enfermería Cardiovascular; Relaciones Comunidad-Institución. 
Fundação e implantação de uma liga acadêmica de enfermagem em cardiologia

\section{INTRODUÇÃO}

O conceito de Ligas Acadêmicas (LAs) é descrito como organizações estudantis sem fins lucrativos, gerenciadas por estudantes, com a supervisão de um docente vinculado à instituição de ensino, que têm como objetivo principal aprimorar o conhecimento em determinadas áreas, tratando-se de uma atividade extracurricular, a qual conecta ensino, pesquisa e extensão. Logo, trata-se de uma forma efetiva de suprir possíveis insuficiências curriculares, visto que conta com aulas teóricas facilitadas por especialistas na área, vivências práticas e pesquisas científicas, o que atrai a atenção de inúmeros estudantes que buscam suprir e aperfeiçoar os conhecimentos na temática em questão (ANDREONI et al., 2019).

No Brasil, a primeira liga acadêmica surgiu em 1920 visando o combate à sífilis, criada na faculdade de medicina da Universidade de São Paulo (USP) por estudantes com intuito de intervir num problema de saúde pública que despontava na época. Com o início do século XXI, mais ligas passaram a ser criadas pelo Brasil, coincidindo com os períodos de reformas curriculares e debates político/acadêmicos sobre os profissionais de saúde a serem formados (FILHO HARMAMOTO, 2011)

Dessa forma, faz-se necessária que as atividades desenvolvidas por LAs tenham relevância acadêmica e social, com ganhos para a população e comunidade científica, devendo ter seus objetivos definidos e articulados entre ensino, pesquisa e extensão. Assim, as LAs se mostram como um instrumento complementar, visando não só aplicar conhecimentos aprendidos, mas gerir mudanças na universidade e comunidade, exigindo um constante trabalho em equipe entre os membros participantes: os ligantes, orientadores e diretores (FILHO HARMAMOTO, 2011 \& YANG, 2019).

Deste modo, as LAs oferecem inúmeros benefícios, não somente para os acadêmicos, mas também para a instituição e para a sociedade, dado que a participação nas mesmas permite ao estudante o conhecimento aprimorado, aquisição de experiência, uma aproximação maior com a sociedade e qualificação profissional (PONTES; TORREÃO, 2019).

As atividades de uma LA voltada à cardiologia, dentro de um hospital de referência, permite a participação de acadêmicos em um cenário de práticas reais, onde estes podem atuar como protagonistas na prevenção e promoção de saúde dos pacientes que recorrem ao hospital, além disso, auxiliam no serviço, integrando teoria e prática, evitando até mesmo a sobrecarga de trabalho dos profissionais envolvidos. Sendo válido ressaltar a relevância da experiência para reflexão crítica, vivenciando diversas situações, com diferentes preceptores e colegas, assistindo novos pontos de vidas, condutas e técnicas (MARQUES et al., 2016). 
Fundação e implantação de uma liga acadêmica de enfermagem em cardiologia

A partir disso, emerge a Liga de Enfermagem em Cardiologia (LECARDIO), derivada da iniciativa de discentes do Curso de Enfermagem da Universidade Estadual Vale do Acaraú (UVA), em Sobral-CE, ao perceberem a necessidade da inserção do estudante de enfermagem nos cuidados à pessoa com doença cardiovascular, visto ser incipiente o conteúdo teórico-prático na grade curricular em geral.

Este estudo é relevante diante da incipiência de artigos publicados acerca de ligas acadêmicas de enfermagem na área da cardiologia, tendo em vista que o levantamento bibliográfico apresentou a existência de diversas ligas dessa temática em graduações de medicina.

Diante do exposto, objetiva-se descrever a fundação e implantação de uma Liga de Enfermagem em Cardiologia.

\section{METODOLOGIA}

Trata-se de um estudo qualitativo, descritivo, do tipo relato de experiência, acerca do processo de fundação e implantação da Liga de Enfermagem em Cardiologia (LECARDIO) da Universidade Estadual Vale do Acaraú (UVA), em Sobral-Ceará.

A LECARDIO/UVA foi idealizada em 2018 por um grupo de quatro graduandos em enfermagem da UVA, denominados diretores discentes e com o suporte docente de uma professora titular da instituição, a qual possui especialização, mestrado e doutorado na área. A liga, atualmente, tem parceria com o Hospital do Coração (HC) de Sobral - Padre José Linhares Ponte, espaço no qual são realizadas extensões e plantões com vistas ao aprofundamento prático dos discentes e sua equipe ampliou-se para dezessete alunos da graduação, após processo seletivo idealizado em 2019; dois docentes e profissionais da instituição hospitalar parceira.

O HC de Sobral é uma instituição Filantrópica anexa à Santa Casa de Misericórdia de Sobral (SCMS), que dispõe de todos os métodos diagnósticos e completares em Cardiologia. É responsável por cobrir toda a macrorregião da Zona Norte do Estado do Ceará, tendo cerca de 85\% dos seus atendimentos pelo Sistema Único de Saúde. Além disso, tem participação em vários estudos multicêntricos nacionais e internacionais na área de Cardiologia, onde mantêm vínculos científicos com importantes instituições do Brasil, Europa e Estados Unidos (SANTA CASA DE MISERICÓRDIA DE SOBRAL, 2012).

A LECARDIO/UVA tem por finalidade promover aos acadêmicos de enfermagem o aprofundamento teórico e prático no âmbito do cuidado de enfermagem aos pacientes com cardiopatias; propiciar momentos de integração entre academia e serviço por meio do ensino, pesquisa e extensão em enfermagem cardiovascular, como meio de aperfeiçoar prática baseada 
Fundação e implantação de uma liga acadêmica de enfermagem em cardiologia

em evidência; e auxiliar a equipe de enfermagem no cuidado aos pacientes com cardiopatias, através de ações de promoção à saúde cardiovascular, acolhimento com classificação de risco e cuidado ao paciente em estado crítico.

A fundação da LECARDIO/UVA deu-se entre os meses de janeiro a junho de 2019, sendo implementada oficialmente em julho do mesmo ano. Todos os trâmites foram documentados pelos discentes membros do corpo diretor da liga e encontram-se descritos a seguir.

\section{RELATO DE EXPERIÊNCIA E ANÁLISE}

A LECARDIO baseia-se no interesse pela cardiologia despertado por vivências pessoais e experiências ao longo da graduação do Curso de Enfermagem da UVA, visando proporcionar uma melhor formação profissional a seus integrantes, causar integração entre acadêmicos e docentes, pesquisadores da saúde cardiovascular, serviço de saúde e população, realizando atividades que contribuam com a sociedade na promoção da saúde e da qualidade de vida. Também visa possibilitar vivenciar as competências e o papel da enfermagem nos cuidados aos pacientes cardiológicos, levando em considerações os princípios do ensino, pesquisa e extensão.

As LAs são um fenômeno ascendente no Brasil, que coincidem com as reformas curriculares e possibilitam uma formação diferenciada em saúde, pois inserem seus participantes nos campos de atuação, possibilitando o preenchimento de lacunas de conhecimento presentes na graduação através do protagonismo e autonomia discente (CAVALCANTE et al., 2018)

A LECARDIO surge com o desejo de alunos de aprofundar os conhecimentos em cardiologia, devido à grade curricular do curso ter poucas disciplinas voltadas especificamente para o estudo dessa temática, existindo uma lacuna a ser preenchida, sendo um tema não estudado especificamente por outras LAs já existentes dentro da universidade ou na região.

Tendo em vista que as doenças cardiovasculares são a maior causa de morte no mundo e são responsáveis por cerca de 30\% dos óbitos no Brasil, além de causarem aumento de internações cirúrgicas cardíacas, por consequência da transição epidemiológica e do envelhecimento da população, surge a necessidade de se ter uma LA com fins de aprofundamento em saúde cardiovascular, tendo em vista a capacitação de acadêmicos dos cursos de saúde para enfrentar essa nova realidade epidemiológica (SIQUEIRA; SIQUEIRA-FILHO; LAND, 2017 \& OKAMOTO et al., 2018).

Nesse ínterim, é fundamental que haja preparo e qualificação da saúde para assistir essa demanda, onde as LAs se tornam projetos que instruem o aluno a fim de que ele se torne um 
agente de transformação social, capacitando profissionais da saúde acerca dos principais problemas epidemiológicos do território que estão inseridos (OKAMOTO et al., 2018).

O processo de formação da liga ocorreu da seguinte forma, conforme se apresenta na Ilustração 1:

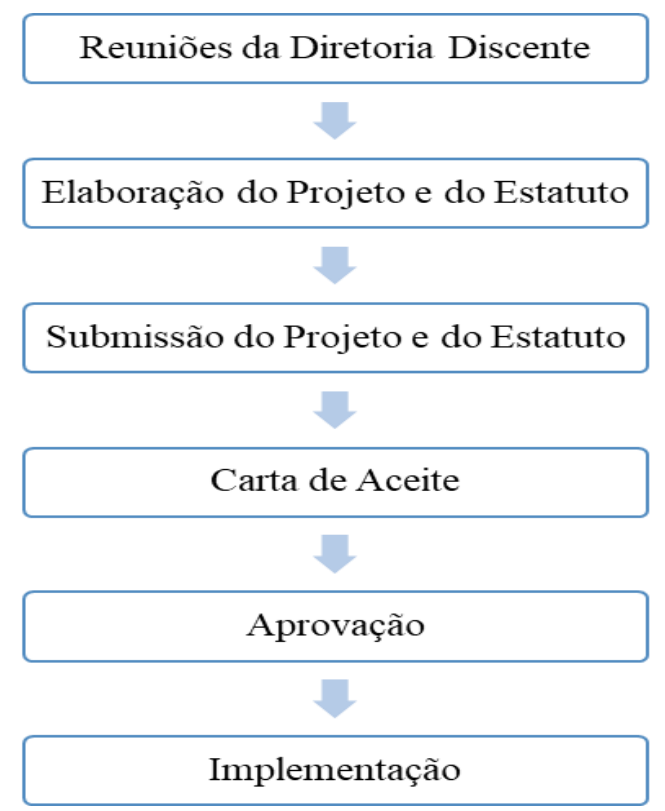

Ilustração 1- Processo de Fundação da LECARDIO, Sobral-CE (2020)

Fonte: Elaborado pelos autores

Inicialmente ocorreu uma reunião dos membros fundadores, com a finalidade de distribuir competências, concretizar o projeto e o estatuto, preencher documentos, traçar os processos burocráticos e os órgãos que necessitariam ser contatados para oficializar a liga. Nesse momento, ocorreu a locação de cada cargo da diretoria que cada membro iria ocupar, de acordo com sua identificação e habilidades, após ser realizada uma dinâmica com cartazes contendo as seguintes perguntas: "Como você se sente em relação à construção da LECARDIO?”, "Quais suas expectativas e de que forma você pode contribuir com a liga?"; "Como você vê a liga em um ano?". Os cartazes passaram por cada membro de forma individual, objetivando a exposição dos seus sentimentos diante da criação de uma LA. Ao final, ficaram estabelecidos os seguintes cargos: presidência; diretoria de pesquisa e extensão; diretoria financeira e diretoria de ensino.

Após a finalização do projeto e do estatuto sob orientação docente, o Hospital do Coração (HC) foi contactado para firmar contrato com a implementação. Ao tempo que se obteve aprovação do HC, entrou-se em contato com a coordenação do curso da instituição em busca de um parecer para regulamentar o projeto, e só então o projeto foi enviado aos órgãos 
regulamentadores de extensão: Departamento de Ensino Pesquisa e Extensão (DEPE), da Santa Casa de Misericórdia de Sobral (SCMS), e para a Pró-reitoria de Extensão e Cultura da UVA.

De acordo com as diretrizes curriculares nacionais do curso de graduação em enfermagem, instituído pelo Conselho Nacional de Educação, o artigo $4^{\circ}$ coloca que o enfermeiro deve adquirir junto à sua formação as seguintes habilidades e competências: devem estar aptos a desenvolver ações preventivas, de promoção, proteção e reabilitação da saúde, tanto em nível individual quanto coletivo, deve estar apto a tomada de decisões, devendo ser capaz de avaliar, sistematizar e decidir condutas baseadas em evidências científicas, devendo também ter capacidade para assumir posições de liderança, tomar iniciativa e administrar recursos, além de terem compromissos com a educação (MINISTÉRIO DA EDUCAÇÃO, 2001).

Com isso, a LA possibilita o desenvolvimento de diversas habilidades e competências exigidas pelas diretrizes curriculares, tornando discentes protagonistas e líderes do processo de aprendizagem e abrindo espaço para adquirir novos saberes.

Para seleção dos ligantes foi lançado um edital, selecionando treze acadêmicos do curso de enfermagem da UVA matriculados regularmente entre o $5^{\circ}$ e o $8^{\circ}$ semestre, visando à disponibilidade e às habilidades necessárias para desenvolver as atividades propostas dentro do campo de extensão. As inscrições foram realizadas através de e-mail, no qual os candidatos enviavam um formulário disponibilizado junto ao edital e o histórico acadêmico atualizado.

A seleção foi dividida em duas etapas, sendo a primeira uma avaliação teórica com questões objetivas, com bibliografia disponibilizada em anexo no edital e a segunda uma entrevista, onde foram analisadas habilidades atitudinais dos inscritos, havendo questionamentos sobre situações que vivenciariam dentro das práticas no hospital. Para classificação dos acadêmicos, as duas notas foram somadas e divididas para resultar numa média final.

Divulgado o resultado da seleção, os acadêmicos tiveram um momento de acolhimento do auditório do HC, onde foram explicados os objetivos da liga e como ocorreria a estruturação para contemplar o ensino, pesquisa e extensão. Posteriormente, foi realizada uma visita técnica para conhecer a estrutura do hospital e seus serviços.

Antes do início dos plantões, os ligantes passaram por dois meses de formações teóricas, tendo dois encontros na semana, segunda e quarta-feira, onde foram abordados os conteúdos dispostos no quadro 01, os quais envolviam assuntos relacionados à enfermagem em cardiologia e o desenvolvimento de pesquisas científicas na área. Ao início dos plantões, ficou estabelecido um encontro por semana, nos quais as temáticas trabalhadas eram sugeridas pelos acadêmicos de acordo com as necessidades oriundas da prática. 


\begin{tabular}{|c|c|}
\hline Encontro & Temática \\
\hline 1 & Acolhida dos ligantes e apresentação da LECARDIO. \\
\hline 2 & Visita técnica às dependências do Hospital do Coração de Sobral. \\
\hline 3 & $\begin{array}{l}\text { Aula: Prevenção de doenças cardiovasculares e Elaboração de tecnologias } \\
\text { educativas. }\end{array}$ \\
\hline 4 & Aula: Fisiologia e anatomia do sistema cardiovascular. \\
\hline 5 & Aula: Parada cardiorrespiratória e Reanimação cardiorrespiratória. \\
\hline 6 & Aula: Semiologia e semiotécnica do sistema cardiovascular. \\
\hline 7 & Aula: Interpretação de Eletrocardiograma. \\
\hline 8 & $\begin{array}{l}\text { Aula: Avaliação do estado de saúde do paciente em Unidade de Terapia Intensiva: } \\
\text { Anamnese e Exame Físico. }\end{array}$ \\
\hline 9 & $\begin{array}{l}\text { Aula: Pesquisa científica na biblioteca virtual em saúde e Elaboração de estudo } \\
\text { metodológico. }\end{array}$ \\
\hline 10 & Aula: Monitorização invasiva e não-invasiva de paciente em unidade coronariana. \\
\hline 11 & Aula: Sistematização da assistência de enfermagem. \\
\hline 12 & Aula: Manipulação de dispositivos invasivos. \\
\hline 13 & $\begin{array}{l}\text { Aula: Unidade de Terapia Intensiva: Organização e processo de trabalho em } \\
\text { enfermagem. }\end{array}$ \\
\hline 14 & Aula: Acolhimento com Classificação de Risco em Pronto Atendimento \\
\hline 15 & Aula: Medicamentos mais utilizados na Unidade de Terapia Intensiva. \\
\hline 16 & $\begin{array}{l}\text { Prática: Construção de tecnologias educativas para a promoção da saúde } \\
\text { cardiovascular. }\end{array}$ \\
\hline 17 & $\begin{array}{l}\text { Sessão Clínica: Discussão de casos clínicos da Unidade Coronariana e Pronto } \\
\text { Atendimento. }\end{array}$ \\
\hline 18 & Aula: Gasometria arterial: Prática e Interpretação. \\
\hline 19 & Aula: Cuidados de enfermagem em hemodinâmica e cardiologia intervencionista. \\
\hline 20 & Aula: Citações e referências bibliográficas em pesquisas científicas. \\
\hline 21 & Aula: Atualização em prevenção e manejo de lesões de pele. \\
\hline 22 & Aula: Eletrocardiografia clínica aplicada à prática. \\
\hline
\end{tabular}

Quadro 1 - Temáticas abordadas nos encontros semanais da liga.

Fonte: Elaborado pelos autores. 
Fundação e implantação de uma liga acadêmica de enfermagem em cardiologia

A equipe da LECARDIO é composta por quatro membros da diretoria, duas docentes da UVA, seis enfermeiros do HC e treze ligantes. Como estratégia de organização das futuras atividades de pesquisa, promoveu-se a organização da equipe em quatros subgrupos de pesquisa, nos quais cada membro da diretoria ficou responsável por um, sendo alocados profissionais do hospital e aproximadamente três ligantes em cada. As duas professoras docentes fazem a supervisão dos quatro grupos. Essa organização permitiu melhor aprofundamento de temáticas específicas para pesquisas científicas.

Quanto às extensões, elas são organizadas em plantões de 12 horas semanais no HC, nas Unidades Coronarianas (UCO) e no Pronto Atendimento (PA), ficando dois ligantes por plantão. Os plantões são realizados em um dos seguintes horários, conforme escala pré-estabelecida pela coordenação discente: $18 \mathrm{~h} 30$ às $06 \mathrm{~h} 30$ (de segunda a sexta) e 06h30 às $18 \mathrm{~h} 30$ (sábados e domingos). Além disso, soma-se à carga horária de extensão a realização de ações de educação em saúde com pacientes das enfermarias mensalmente.

Outras atividades desenvolvidas pela LECARDIO são as sessões clínicas, nas quais são discutidos casos clínicos específicos sugeridos pelos ligantes. Também houve a criação de tecnologias educativas (TEs) para serem aplicadas durante a realização de ações de educação em saúde, sendo construídas por subgrupos de pesquisa.

As TEs desenvolvidas foram "ReabilitaCardio", "DadoCardio", "MemóriaCardio e "Baralhocardio". O "ReabilitaCardio" trata-se de um jogo semelhante à batalha naval, onde há uma sequência de números de 1 a 5 na borda vertical, e na horizontal as letras A, B e C. A TE contém figuras de corações saudáveis e de bombas, onde o condutor do jogo explana sobre condutas favoráveis para reestabelecer a saúde do paciente internado e hábitos que não se deve ter, que podem prejudicar a recuperação.

O "DadoCardio" trata-se de uma TE em formato de dado que contém envelopes em cada lado, com perguntas de verdadeiro ou falso acerca dos fatores de risco modificáveis para o desenvolvimento de doenças cardiovasculares. O paciente deverá jogar o dado e retirar uma pergunta do envelope correspondente ao lado que parou. Abordam-se conteúdos como sedentarismo, obesidade, tabagismo e hipertensão arterial e, a partir das respostas dos pacientes, o condutor orienta hábitos saudáveis de vida.

O "MemóriaCardio" trata-se de um jogo da memória com 12 pares de imagens representando os fatores de riscos e os hábitos favoráveis para a prevenção do adoecimento cardiovascular, do qual podem participar de 2 a 6 pessoas, reunidas em um círculo, e a cada par de figuras encontrado os participantes são questionados sobre o que a imagem representam - se 
trata de algo benéfico ou maléfico à saúde cardiovascular e o porquê, e a partir das respostas o condutor realiza orientações sobre esses hábitos.

O "BaralhoCardio" é composta por 36 cartas, sendo uma metade apresentando imagens que representam problemas cardiovasculares e a outra com propostas de intervenção. As cartas com problemas cardiovasculares ficam em posse dos facilitadores do jogo, enquanto as cartas com frases de intervenções são distribuídas aleatoriamente aos participantes, sendo apresentadas as cartas dos problemas de forma a solicitar que os participantes apresentem as intervenções, a partir das cartas em posse.

Cada uma das TEs serão aplicadas no contexto hospitalar de pacientes internados na enfermaria do HC, além de ser possível sua utilização em outros espaços, a depender das necessidades de extensão da LECARDIO.

A utilização de TEs permite que haja uma interação maior entre os participantes, o que facilita o aprendizado, tornando a educação em saúde mais interessante e eficaz, visto que proporciona o diálogo, a construção do conhecimento em grupo e a partir disso torna os participantes capazes de eles mesmos propagarem as informações obtidas de forma simples. Sendo assim um artifício valioso, atrativo e de baixo custo que tem como consequência a compreensão dos participantes do assunto abordado e uma liberdade maior para fazer questionamentos que durante uma consulta sentem-se tímidos para perguntar (GURGEL et al., 2017).

Importante ressaltar que houve dificuldades durante o processo de implementação da liga, tendo em vista a necessidade do desenvolvimento de um projeto sólido que fosse ao encontro das necessidades tanto da universidade, quanto do local em que aconteceriam as extensões. Contudo, os acadêmicos puderam contar com o apoio e com a motivação das docentes responsáveis pela liga, o que contribuiu para a idealização dos processos.

Partindo desses ideais, compreende-se que as atividades extracurriculares, em específico as LAs, garantem complemento à formação profissional, dão autonomia, suprem a necessidade do contato do acadêmico com a prática profissional, possibilitam a exploração de áreas de atuação na profissão e o desenvolvimento de habilidades. Além disso, as experiências extracurriculares são convertidas em aptidão para inserção no mercado de trabalho, onde o exercício não contribui apenas para elaboração de currículos dos formandos, mas também para a autoeficácia profissional. Podem também auxiliar em atendimentos individuais para alunos insatisfeitos com o curso, amparando a decisão de permanência ou evasão do curso de graduação dos alunos que se queixam do distanciamento entre os conteúdos teóricos em aulas e a profissão (OLIVEIRA; SANTOS; DIAS, 2016). 


\section{CONSIDERAÇÕES FINAIS}

A LECARDIO foi idealizada por discentes de enfermagem; fundada por esses discentes, sob orientação docente e de profissionais de enfermagem de uma instituição hospitalar de cardiologia e sua implementação realizada a partir da aprovação por órgãos regulamentadores de extensão, após a confecção de documentos oficiais - estatuto e projeto de extensão.

A liga se trata de um projeto que envolve ensino, pesquisa e extensão. As atividades de ensino são formações teóricas sobre enfermagem em cardiologia e pesquisa científica, as extensões englobam plantões no Hospital do Coração de Sobral e atividades de educação em saúde através de tecnologias educativas, enquanto as pesquisas serão desenvolvidas por intermédio da subdivisão da equipe da LECARDIO em quatro grupos.

A criação e implementação da LECARDIO possibilitou aos acadêmicos autonomia, devido à possibilidade de aprofundamento em uma área não muito explorada pela grade curricular do curso, garantindo o desenvolvimento de competências de enfrentamento, como responsabilidade, liderança, execução do trabalho em grupo e gerenciamento frente a uma atividade extracurricular que permite atende ao tripé: ensino-pesquisa-extensão.

Desta forma, é possível concluir que a criação de LAs contribui de forma significativa no ensino, visto que agrega novos conhecimentos para os acadêmicos que vão muitas vezes além da grade curricular. Também para a extensão, devido às inúmeras experiências que proporcionam aos acadêmicos, que são muito mais intensas que os estágios por conta da carga horária superior. E a pesquisa também é beneficiada em virtude da proximidade com o campo de pesquisa e das inúmeras experiências que poderão ser publicadas. E, com certeza, a população será muito beneficiada, posto que usufruirão de mais recurso humano, já que, além dos profissionais do serviço, poderá contar com os estudantes, bem como com as ações de promoção da saúde que são direcionadas à população, a fim de prevenir doenças, e assim contribuir com o desenvolvimento da profissão, o que fortalece a classe de enfermeiros.

Os principais desafios do processo de fundação e implementação da LECARDIO envolveram a articulação entre docentes, discentes e funcionário da instituição hospitalar, pois foi difícil promover encontros presenciais para a organização e alinhamento das atividades. Os próximos passos da LECARDIO são: desenvolver projetos de pesquisa para estudos específicos de enfermagem em cardiologia, nova seleção de ligantes para compor a equipe e expansão das atividades de extensão para outros espaços comunitários. 
Fundação e implantação de uma liga acadêmica de enfermagem em cardiologia

Espera-se que o presente estudo estimule o processo de criação de projetos acadêmicos na área da saúde cardiovascular, como meio de aprimorar os futuros enfermeiros para o cuidado a pacientes cardiopatas e promoção da saúde cardiovascular na comunidade.

\section{REFERÊNCIAS}

ANDREONI, S.; RANGEL, D. C.; BARRETO, G. C. B. G. S.; RODRIGUES, R.H.I.; ALVES, H.M.T.; PORTELA, L.A. O perfil das ligas acadêmicas de angiologia e cirurgia vascular e sua eficácia no ensino da especialidade. J. Vasc. Bras., Porto Alegre, v. 18, e20180063, fev.2019. Disponível em: http://www.scielo.br/pdf/jvb/v18/1677-5449-jvb-18-e20180063.pdf. Acesso em: 29 fev. 2020

CAVALCANTE, A. S. P.; VASCONCELOS, M. I. O.; LIRA, G. V.; HENRIQUES, R. L. M.; ALBUQUERQUE, I. N. M.; MACIEL, G. P.; RIBEIRO, G. P.; GOMES, D. F. As ligas acadêmicas na área da saúde: lacunas do conhecimento na produção científica brasileira. Rev. Brasileira de educação médica, Brasília, v.42, n.1, p. 194-204, jan/mar. 2018. Disponível em: http:/ /www.scielo.br/scielo.php?script=sci_abstract\&pid=S010055022018000100199\&lng=en\& $\mathrm{nrm}=$ iso\&tlng=pt. Acesso em: 20 mai. 2020.

GURGEL, S.S.; TAVEIRA, G.P.; MATIAS, E.O.; PINHEIRO, P.N.C.; VIEIRA, N.F.C.; LIMA, F.E.T. Jogos educativos: recursos didáticos utilizados na monitoria de educação em saúde. REME - Rev Min Enferm, Belo Horizonte, v.21, e-1016, jun 2017. Disponível em: http://www.reme.org.br/artigo/detalhes/1152. Acesso em: 11 abr. 2020.

HAMAMOTO FILHO, P.T. Ligas Acadêmicas: motivações e críticas a propósito de um repensar necessário. Ensaio - Rev. bras. educ. med., v. 35, n. 4, dez 2011. Disponível em: https://www.scielo.br/j/rbem/a/RcH7qnHW8tnC6hvM8kJGHWb/?lang=pt. Acesso em: 20 abr. 2020.

MARQUES, A. C. F.; ROGRIGUES, C. S.; SANTOS, J. A. D.; BRANT, L.C.; SANT'ANA, L.N.M.; NOGUEIRA, H.S.; MEDEIROS, E.E.C.; TELES, C.B. Liga acadêmica de cardiologia: instrumento de formação em medicina. Revista Intercâmbio, Minas Gerais, v. 7, p. 496-503, 2016. Disponível em: http://www.intercambio.unimontes.br/index.php/intercambio/article/view/121/109. Acesso em: 20 mai. 2020.

MINISTÉRIO DA EDUCAÇÃO (BR), Resolução CNE/CNS No3 de 7 de novembro de 2001. Diretrizes Curriculares Nacionais do Curso de Graduação em Enfermagem [Internet]. Brasília: Ministério da Educação; 2001.

OKAMOTO, K. M.; BENASSI, G.; PILATTI, L. D. S.; SCHEIDT, B. J.; LUZ, B. Z.; SANTOS, THERENCY. K.; BOTH, A. S.; COSTA, M. A. C. Liga acadêmica de clínica e cirurgia cardíaca: relato de experiência. Extensio: R. Eletr. de Extensão, Florianópolis, v. 15, n. 30, p. 56-65, 2018. Disponível em: https://periodicos.ufsc.br/index.php/extensio/article/view/18070221.2018v15n30p56/37778. Acesso em: 20 mai. 2020. 
Fundação e implantação de uma liga acadêmica de enfermagem em cardiologia

OLIVEIRA, C. T.; SANTOS, A. S.; DIAS, A. C. G. Percepções de Estudantes Universitários sobre a Realização de Atividades Extracurriculares na Graduação, Psicologia Ciência e Profissão, Brasília, v. 36, n. 4, p.864-876, out./dez. 2016. Disponível em: http://www.scielo.br/pdf/pcp/v36n4/1982-3703-pcp-36-4-0864.pdf. Acesso em: 20 mai. 2020

PONTES, S.; TORREÃO, L. Influência da participação de estudantes em ligas acadêmicas na escolha da especialidade para o programa de residência médica da Bahia 2017. Revista de Medicina, São Paulo, v. 98, n. 3, p. 160-167, 22 jul. 2019. Disponível em: http://www.revistas.usp.br/revistadc/article/view/155902/154512. Acesso em: 20 mai. 2020.

SANTA CASA DE MISERICÓRDIA DE SOBRAL. Hospital do Coração. Disponível em: http://www.stacasa.com.br/hospital-do-coracao-de-sobral-2/. Acesso em: 20 mai. 2020.

SIQUEIRA, A. S. E.; SIQUEIRA-FILHO, A. G.; LAND, M. G. P. Análise do Impacto Econômico das Doenças Cardiovasculares nos Últimos Cinco Anos no Brasil, Arq. Bras. Cardiol., São Paulo, v. 1, n. 109, p.39.46, julho. 2017. Disponível em: http://www.scielo.br/pdf/abc/v109n1/pt_0066-782X-abc-20170068.pdf. Acesso em: 19 mai. 2020.

YANG, G. Y. H. Y.; BRAGA, A. C. B.; HIPÓLITO, N. C.; VIEIRA, K.S.T.; PESSANHA, C.G.; ABRANTES, F.G.; PEREIRA, P.S.; CORRÊA, C.L. Liga de Anatomia Aplicada (LAA): as Múltiplas Perspectivas sobre Participar de uma Liga Acadêmica. Rev. bras. educ. med., Brasília, v. 43, n. 1, p. 80-86, Mar. 2019. Disponível em: http://www.scielo.br/pdf/rbem/v43n1/19815271-rbem-43-1-0080.pdf. Acesso em: 15 fev. 2020.

Recebido em: 24/04/2020

Aceito em: 24/11/2021 